\title{
Robust Static and Dynamic Output-Feedback Stabilization: Deterministic and Stochastic Perspectives
}

\author{
DENNIS S. BERNSTEIN, MEMBER, IEEE
}

\begin{abstract}
Three parallel gaps in robust feedback control theory are examined: sufficiency versus necessity, deterministic versus stochastic uncertainty modeling, and stability versus performance. Deterministic and stochastic output-feedback control problems are considered with both static and dynamic controllers. The static and dynamic robust stabilization problems involve deterministically modeled bounded but unknown measurable time-varying parameter variations, while the static and dynamic stochastic optimal control problems feature state-, control-, and measurement-dependent white noise. General sufficiency conditions for the deterministic problems are obtained using Lyapunov's direct method, while necessary conditions for the stochastic problems are derived as a consequence of minimizing a quadratic performance criterion. The sufficiency tests are then applied to the necessary conditions to determine when solutions of the stochastic optimization problems also solve the deterministic robust stability problems. As an additional application of the deterministic result, the modified Riccati equation approach of Petersen and Hollot is generalized in the static case and extended to dynamic compensation.
\end{abstract}

\section{INTRODUCTION}

$\mathrm{T}$ HE gain and phase margins of full-state-feedback LQ regulators are well known [1], [2]. Although dynamic output-feedback LQG controllers lack such margins [3], considerable effort has been devoted to recovering the full-state-feedback properties [4]-[6]. A crucial point discussed in [7]-[9] is that such margins may be meaningless for guaranteeing robustness with respect to arbitrary plant parameter variations. This was demonstrated by means of a simple example in [7]. In addition, as is well known, the use of singular value bounds to characterize plant uncertainty contributes directly to conservatism with respect to real-valued structured parameter variations.

For the parametric-uncertainty stability robustness problem, there exists a considerable body of literature (see, e.g., [10][25]). These results often rely upon Lyapunov's direct method and thus are usually in the form of sufficient conditions. Two factors are often lacking, however: a measure of performance beyond stability and design considerations involving controller effort. ${ }^{\prime}$

Performance and controller effort are, of course, the natural domain of stochastic optimal control via the cost criterion. In addition, parameter uncertainties can be directly incorporated into the stochastic model by means of multiplicative white noise [26][40]. Heuristically speaking, the performance of a quadratically optimal feedback controller designed in the presence of such

Manuscript received April 23, 1986; revised April 13, 1987. Paper recommended by Associate Editor, D. P. Looze. This work was supported in part by the Air Force Office of Scientific Research under Contracts F4962086-C-0002 and F49620-86-C-0038.

The author is with the Government Aerospace Systems Division, Melbourne, FL 32902.

IEEE Log Number 8716818.

Several exceptions to these remarks should be noted: necessary and sufficient conditions have been given in [12], [21]; a quadratic cost functional is utilized in [10], [13]; and controller effort is considered in [20]. multiplicative disturbances is desensitized to actual constant or time-varying parameter variations. It should be emphasized that the white noise parameter-uncertainty model is not interpreted literally as a physical model. Rather, the multiplicative noise model serves as a device which captures the effect of parameter uncertainty on the second-moment matrix, and hence on the closed-loop performance. From a practical point of view, the multiplicative white noise model is extremely tractable since the second-moment equation is closed and the optimal feedback gains can be given explicitly by closed-form expressions involving solutions of algebraic equations. For example, the necessary conditions derived in [40] for quadratically optimal steady-state fixed-order (i.e., reduced-order) dynamic compensation in the presence of state-, control-, and measurement-dependent white noise involve a coupled system of two modified algebraic Riccati equations and two modified algebraic Lyapunov equations. The coupling is due to both the optimal projection, which enforces the fixed-order constraint [41], and the multiplicative white noise terms. Unfortunately, however, stochastic optimal control is predicated upon stability of the second moment of the state [42][47], which may be weaker than deterministic robust asymptotic stability. As a matter of fact, it has been shown, rather surprisingly, that a nominally unstable system can be rendered stochastically stable by multiplicative white noise interpreted in the sense of Stratonovich without actually applying feedback control [48]. ${ }^{2}$ Hence, there is no prior guarantee that a secondmoment stable optimal controller predicated upon a multiplicative white noise model will provide deterministic robust or even nominal asymptotic stability.

Three parallel gaps can thus be perceived between the above approaches: sufficiency versus necessity, deterministic versus stochastic uncertainty modeling, and stability versus performance. In attempting to bridge these gaps we ask the following question: When is the solution to a stochastic optimal control problem also the solution to a deterministic robust stabilization problem? In the present paper we show that our necessary conditions for stochastic optimality become sufficient conditions for deterministic robustness when we include an exponential weighting factor in the quadratic cost criterion. As shown in [49], the weighting factor $e^{2 \alpha t}$ leads to replacement of the closed-loop dynamics matrices

$$
A+B K C,\left[\begin{array}{cc}
A & B C_{c} \\
B_{c} C & A_{c}
\end{array}\right]
$$

for static and dynamic controllers, respectively, by the shifted dynamics matrices

$$
A+\alpha I_{n}+B K C,\left[\begin{array}{cc}
A+\alpha I_{n} & B C_{c} \\
B_{c} C & A_{c}+\alpha I_{n_{c}}
\end{array}\right] .
$$

When there are no parametric plant uncertainties, a right shift ( $\alpha$

\footnotetext{
2 This phenomenon does not occur, however, with the Ito interpretation.
} 
$>0)$ yields a prescribed stability margin, i.e., all closed-loop poles having real part less than $-\alpha$. Unfortunately, a right shift alone does not appear to provide guaranteed stability robustness levels with respect to arbitrary plant variations. Since multiplicative white noise also does not ensure robustness, the present paper goes beyond previous work by employing the right shift in conjunction with multiplicative white noise to guarantee robust stability over a specified range of deterministic parameter variations. In particular, we consider perturbations of $A, B$, and $C$ of the form

$$
A+\sum_{i=1}^{p} \sigma_{i}(t) A_{i}, B+\sum_{i=1}^{p} \sigma_{i}(t) B_{i}, C+\sum_{i=1}^{p} \sigma_{i}(t) C_{i},
$$

where $A_{i}, B_{i}$, and $C_{i}$ denote the pattern of parametric uncertainty in $A, B$, and $C$ and $\sigma_{i}(\cdot)$ are real-valued Lebesgue measurable functions satisfying

$$
\left|\sigma_{i}(t)\right| \leq \delta_{i}, \quad i=1, \cdots, p
$$

In this formulation the patterns $A_{i}, B_{i}$, and $C_{i}$ are assumed to be known while the deterministically modeled uncertain parameters $\sigma_{i}(\cdot)$ are known except for the bounds (1.1). Our principal result for both static and dynamic controllers states that a solution of the necessary conditions for stochastic optimal control with exponential weighting and multiplicative white noise provides guaranteed robust asymptotic stability for parametric variations satisfying (1.1) as long as

$$
\alpha>\frac{1}{2} \sum_{i=1}^{p} \delta_{i}^{2} / \gamma_{i}
$$

where $\alpha$ is the right shift and $\gamma_{i}$ is the intensity of the noise $v_{i}(t)$ multiplying $A_{i}, B_{i}$, and $C_{i}$ in the multiplicative white noise formulation of the stochastic optimal control problem. Clearly, the rectangular robust stability region $\left[-\delta_{1}, \delta_{1}\right] \times \cdots \times\left[-\delta_{p}\right.$, $\left.\delta_{p}\right]$ in $p$-dimensional parameter space can be enlarged by increasing either $\alpha$ or $\gamma_{1}, \cdots, \gamma_{p}$. Note that for given values of $\alpha$, $\gamma_{1}, \cdots, \gamma_{p}(1.2)$ does not define a unique robustness region when $p>1$. The robust stability guarantee holds, however, for simultaneous parameter variations $\sigma_{1}(\cdot), \cdots, \sigma_{p}(\cdot)$ within each region satisfying (1.2).

The above result is based upon the observation that secondmoment stability of a stochastic system with multiplicative disturbances and right-shifted mean dynamics induces a Lyapunov function which guarantees robust stability of a deterministic system subject to time-varying parameter variations. This observation, which appears to have been previously overlooked in the literature, may be utilized in the context of robustness analysis for linear uncertain systems. In the present paper this result is developed within the context of robust controller synthesis to achieve a unified approach to robust, fixed-order controller design consistent with [41].

The derivation of our results is quite simple and is based upon the standard quadratic Lyapunov function

$$
V(x)=x^{T} P x
$$

where $P$ is given by the modified Lyapunov equation

$$
0=\tilde{A^{T} P}+P \tilde{A}+2 \alpha P+\sum_{i=1}^{p} \gamma_{i} A_{i}^{T} P A_{i}+\tilde{R}
$$

where $\tilde{A}$ is the closed-loop dynamics matrix and $\tilde{R}$ is a closedloop weighting matrix. Note that the third and fourth terms in (1.4) correspond to exponential weighting and multiplicative white noise, respectively. The result that $\dot{V}(x) \leq-\gamma\|x\|^{2}$, with $\gamma>0$, follows directly from the inequality

$$
\sum_{i=1}^{p} \sigma_{i}(t)\left(A_{i}^{T} P+P A_{i}\right) \leq \sum_{i=1}^{p}\left(\delta_{i}^{2} / \gamma_{i}\right) P+\sum_{i=1}^{p} \gamma_{i} A_{i}^{T} P A_{i}
$$

along with (1.2). Inequality (1.5) follows immediately from

$$
\sum_{i=1}^{p}\left[\gamma_{i}^{1 / 2} A_{i}-\left(\sigma_{i}(t) / \gamma_{i}^{1 / 2}\right) I_{n}\right]^{T} P\left[\gamma_{i}^{1 / 2} A_{i}-\left(\sigma_{i}(t) / \gamma_{i}^{1 / 2}\right) I_{n}\right] \geq 0
$$

and $\sigma_{i}^{2}(t) \leq \delta_{i}^{2}$.

An alternative approach to guaranteeing robustness of designs predicated upon a multiplicative white noise model is to interpret the stochastic differential equation according to Stratonovich stochastic integration instead of Ito integration [36], [37]. Now the closed-loop dynamics matrices become

$$
A_{s}+B_{s} K C,\left(\begin{array}{cc}
A_{s} & B_{s} C_{c} \\
B_{c} C_{s} & A_{c}
\end{array}\right)
$$

where

$$
A_{s}=A+\frac{1}{2} \sum_{i=1}^{p} \gamma_{i} A_{i}^{2}, B_{s}=B+\frac{1}{2} \sum_{i=1}^{p} \gamma_{i} A_{i} B_{i}, C_{s}=C+\frac{1}{2} \sum_{i=1}^{p} \gamma_{i} C_{i} A_{i}
$$

The closed-loop Stratonovich correction evident in $A_{s}, B_{s}$, and $C_{s}$, which can negate the so-called "uncertainty threshold principle" [50], [51], appears to be crucial for designing vibration-suppression controllers for flexible structures [37], [38], [52]. Because of inherent damping, such systems are usually nominally open-loop stable with nondestabilizing uncertainties so that robust stability is less of a challenge than robust performance. Although conditions under which the Stratonovich model yields robust controllers are beyond the scope of the present paper, it should be noted that the differences between the two models are far from trivial. For example, for frequency uncertainties the Stratonovich correction, which corresponds to a variable left shift rather than a uniform right shift, automatically induces a positivereal controller for the high-frequency, poorly modeled flexible modes. Since quadratic Lyapunov functions do not appear to be adequate for guaranteeing the robustness of such designs, the majorant Lyapunov function has been developed [53].

Inasmuch as deterministic robust stability of stochastically optimal controllers is guaranteed by right shift/multiplicative white noise modifications to the closed-loop Lyapunov equation, a natural question which arises is the following: Do there exist alternative modifications to the closed-loop Lyapunov equation which guarantee robust stability? One possibility which immediately suggests itself is to replace the bound (1.5) by

$$
\sum_{i=1}^{p} \sigma_{i}(t)\left(A_{i}^{T} P+P A_{i}\right) \leq \sum_{i=1}^{p} \delta_{i}\left(P D_{i} D_{i}^{T} P+E_{i}^{T} E_{i}\right)
$$

where $A_{i}=D_{i} E_{i}$. Carrying out full-state-feedback control design with (1.7) leads to an alternative generalization of the standard algebraic regulator Riccati equation. Indeed, a version of this modified Riccati equation has already been developed by Petersen and Hollot [23], [25] as a means for designing robust static and dynamic controllers. A fourth-order aircraft example considered in [25] shows the practical potential of their approach. In the present paper we extend the results of [23], [25] to more general problem formulations encompassing a wider class of parametric uncertainty structures within the context of static output feedback and reduced-order dynamic compensation. Most interestingly, the results we obtain are completely analogous to the static and 
dynamic compensation results obtained using a multiplicative white noise model with quadratic cost. This raises the following interesting question: Does there exist an optimization problem whose necessary conditions coincide with the Petersen-Hollottype equations? Indeed, our results were obtained by optimizing over the class of closed-loop Lyapunov equations modified (i.e., robustified) in the sense of Petersen and Hollot. Full justification for this technique is developed in [54], [55] where robust performance bounds are obtained.

\section{Notation AND Definttions}

Note: all matrices have real entries.

$\mathbb{R}, \mathbb{R}^{r \times s}, \mathbb{R}^{r}$
$I_{r},()^{T}$
$\oplus, \otimes$
$\mathbb{S}^{r}$
$\mathbb{N}^{r}$
$\mathbb{P}^{r}$
$Z_{1} \geq Z_{2}$
$Z_{1}>Z_{2}$
asymptotically stable
$\quad$ matrix
$n, m, l, p, n_{c}, n_{i}$,
$\tilde{n}, \tilde{n}_{i}$
$x, u, y, x_{c}$
$A, A_{i} ; B, B_{i} ; C, C_{i}$
$K$
$\tilde{A}, \tilde{A_{i}}$
$A_{c}, B_{c}, C_{c}$
$\tilde{\tilde{A}}, \tilde{A_{i}}$

real numbers, $r \times s$ real matrices, $\mathbb{R}^{r \times 1}$ $r \times r$ identity matrix, transpose Kronecker sum, Kronecker product [56] $r \times r$ symmetric matrices $r \times r$ symmetric nonnegative-definite matrices $r \times r$ symmetric positive-definite matrices $Z_{1}-Z_{2} \in \mathbb{N}^{r}, Z_{1}, Z_{2} \in \mathbb{S}^{r}$ $Z_{1}-Z_{2} \in \rho^{r}, Z_{1}, Z_{2} \in \mathbb{S}^{r}$ matrix with eigenvalues in open left-half plane

positive integers, $i=1, \cdots, p$

$n+n_{c}, n+n_{i}, i=1, \cdots, p$

$n, m, l, n_{c}$-dimensional vectors

$n \times n$ matrices; $n \times m$ matrices, $l \times n$ matrices; $i=1, \cdots, p$

$m \times l$ matrix

$A+B K C, A_{i}+B_{i} K C, i=1, \cdots, p$ $n_{c} \times n_{c}, n_{c} \times l, m \times n_{c}$ matrices

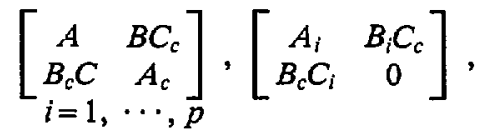

$\sigma_{i}(\cdot)$

$\delta_{i}$

${ }_{A_{\alpha}}, \tilde{A_{\alpha}}, \tilde{\tilde{A}}_{\alpha}$

$R_{1}$

$R_{2}$

$R_{12}$

$\tilde{R}$

$\check{\tilde{R}}$

$w_{t}$

$w_{1 t}$

$w_{2 t}$

$V_{1}$

$V_{2}$

$V_{12}$

$V$

$v_{i t}$

$\gamma_{i}$

㝍

processes, $i=1, \cdots, p$ incremental covariance of $v_{i t}, \gamma_{i}>0$, $i=1, \cdots, p$

expected value
For the following definitions, let $Q, P, \hat{Q}, \hat{P} \in \mathbb{R}^{n \times n}$ :

$$
\begin{gathered}
R_{2 s} \triangleq R_{2}+\sum_{i=1}^{p} \gamma_{i} B_{i}^{T} P B_{i}, \\
P_{s} \triangleq B^{T} P+R_{12}^{T}+\sum_{i=1}^{p} \gamma_{i} B_{i}^{T} P A_{i}, \\
R_{2 d} \triangleq R_{2}+\sum_{i=1}^{p} \gamma_{i} B_{i}^{T}(P+\hat{P}) B_{i},
\end{gathered}
$$

$$
\begin{gathered}
P_{d} \triangleq B^{T} P+R_{12}^{T}+\sum_{i=1}^{p} \gamma_{i} B_{i}^{T}(P+\hat{P}) A_{i}, \\
V_{2 d} \triangleq V_{2}+\sum_{i=1}^{p} \gamma_{i} C_{i}(Q+\hat{Q}) C_{i}^{T}, \\
Q_{d} \triangleq Q C^{T}+V_{12}+\sum_{i=1}^{p} \gamma_{i} A_{i}(Q+\hat{Q}) C_{i}^{T}, \\
A_{P \alpha} \triangleq A_{\alpha}-B R_{2 d}^{-1} P_{d}, \\
A_{Q \alpha} \triangleq A_{\alpha}-Q_{d} V_{2 d}^{-1} C .
\end{gathered}
$$

\section{Static OUtPut FeEdBaCK}

\section{A. Static Robust Stabilization Problem: Deterministic Sufficiency Theory}

Consider the following problem.

Static Robust Stabilization Problem: Determine $K \in \mathbb{R}^{m \times l}$ such that the closed-loop system consisting of the controlled plant

$$
\begin{aligned}
\dot{x}(t)=\left(A+\sum_{i=1}^{p} \sigma_{i}(t) A_{i}\right) x(t) & \\
& +\left(B+\sum_{i=1}^{p} \sigma_{i}(t) B_{i}\right) u(t), \quad t \in[0, \infty),
\end{aligned}
$$

measurements

$$
y(t)=C x(t),
$$

and static output-feedback law

$$
u(t)=K y(t)
$$

is asymptotically stable for all measurable $\left(\sigma_{1}, \cdots, \sigma_{p}\right):[0, \infty) \rightarrow$ $\mathrm{R}^{p}$ satisfying

$$
\left|\sigma_{i}(t)\right| \leq \delta_{i}, t \in[0, \infty), i=1, \cdots, p .
$$

Remark 3.1.1: The nominal stabilization problem, i.e., the case in which parameter uncertainties are absent, can be recovered by setting $A_{i}=0$ and $B_{i}=0$. All of the results in this paper can readily be specialized to this case. For brevity, however, the details are omitted.

Remark 3.1.2: The symmetric bounds (3.1.4) are for convenience only. The constraints

$$
\rho_{i} \leq \sigma_{i}(t) \leq \delta_{i}, t \in[0, \infty), i=1, \cdots, p
$$

can be recast in the form (3.1.4) by redefining $A$ and $B$. Further notational simplification is possible by scaling $A_{i}$ and $B_{i}$ so that $\delta_{i}$ 
$=1, i=1, \cdots, p$. For clarity, however, we choose not to do this.

Remark 3.1.3: Standard existence theory guarantees that the absolutely continuous solution $x(\cdot)$ of $(3.1 .1)$ exists on $[0, \infty)$ for all $K \in \mathbb{F}^{m \times l}$ and for all measurable $\left(\sigma_{1}, \cdots, \sigma_{p}\right)$ satisfying (3.1.4).

The robustness results are based upon the following easily proved theorem which concerns the construction of a Lyapunov function for robust stability.

Theorem 3.1.1: Let $K \in \mathrm{R}^{m \times l}$ and assume there exist

$$
\begin{gathered}
\Phi: \mathbb{P}^{n} \rightarrow \mathbb{S}^{n}, \\
P \in \mathbb{P}^{n}, \\
M_{i}, N_{i} \in \mathbb{P}^{n_{i} \times n}, \quad i=1, \cdots, p
\end{gathered}
$$

such that

$$
\begin{gathered}
M_{i}^{T} N_{i}+N_{i}^{T} M_{i}=\tilde{A_{i}^{T} P+P \tilde{A}}, \quad i=1, \cdots, p \\
0=\tilde{A}^{T} P+P \tilde{A}+\Phi(P) \\
\sum_{i=1}^{p} \delta_{i}\left(M_{i}^{T} M_{i}+N_{i}^{T} N_{i}\right)<\Phi(P) .
\end{gathered}
$$

Then $K$ solves the static robust stabilization problem.

Proof: Using (3.1.8), compute for $t \in[0, \infty)$ and $i=1$, $\cdots, p$,

$$
\begin{aligned}
0 & \leq\left[\delta_{i}^{1 / 2} M_{i}-\delta_{i}^{-1 / 2} \sigma_{i}(t) N_{i}\right]^{T}\left[\delta_{i}^{1 / 2} M_{i}-\delta_{i}^{-1 / 2} \sigma_{i}(t) N_{i}\right] \\
& =\delta_{i} M_{i}^{T} M_{i}+\delta_{i}^{-1} \sigma_{i}^{2}(t) N_{i}^{T} N_{i}-\sigma_{i}(t)\left[M_{i}^{T} N_{i}+N_{i}^{T} M_{i}\right] \\
& \leq \delta_{i}\left[M_{i}^{T} M_{i}+N_{i}^{T} N_{i}\right]-\sigma_{i}(t)\left[\tilde{A}{ }_{i}^{T} P+P \tilde{A}_{i}\right]
\end{aligned}
$$

Thus,

$$
\begin{aligned}
\sigma_{i}(t)\left[\tilde{A}_{i}^{T} P+P \tilde{A}_{i}\right] \leq \delta_{i}\left(M_{i}^{T} M_{i}+N_{i}^{T} N_{i}\right), \\
\quad t \in[0, \infty), i=1, \cdots, p .
\end{aligned}
$$

Defining the Lyapunov function

$$
V(x) \triangleq x^{T} P x
$$

its derivative along solutions $x(t)$ of (3.1.1)-(3.1.3) is given by

$$
\begin{aligned}
\dot{V}(x(t))= & \dot{x}(t)^{T} P x(t)+x(t)^{T} P \dot{x}(t) \\
= & x(t)^{T}\left[\tilde{A}^{T} P+P \tilde{A}\right] x(t) \\
& +x(t)^{T}\left[\sum_{i=1}^{p} \sigma_{i}(t)\left(\tilde{A}_{i}^{T} P+P \tilde{A}_{i}\right)\right] x(t) .
\end{aligned}
$$

Using (3.1.9) and (3.1.11) yields

$$
\dot{V}(x(t)) \leq-x(t)^{T}\left[\Phi(P)-\sum_{i=1}^{p} \delta_{i}\left(M_{i}^{T} M_{i}+N_{i}^{T} N_{i}\right)\right] x(t) .
$$

By (3.1.10), there exists $\gamma>0$ such that $\dot{V}(x(t)) \leq-\gamma\|x(t)\|^{2}$, $t \in[0, \infty)$, as required.

Remark 3.1.4: As will be seen in later sections, this result is applied by choosing $M_{i}$ and $N_{i}$ to satisfy

$$
N_{i}^{T} M_{i}=P \tilde{A_{i}}
$$

so that (3.1.8) holds. The bound $\Phi$ is then constructed to satisfy (3.1.10).

\section{B. Static Optimal Control Problem: Stochastic Necessity Theory}

We now turn to the static optimal control problem with statedependent and control-dependent white noise and exponentially weighted quadratic cost.

Static Optimal Control Problem: Determine $K \in \mathbb{R}^{m \times l}$ such that, for the closed-loop system consisting of the controlled plant

$$
\begin{aligned}
d x_{t}=A x_{t} d t+\sum_{i=1}^{p} A_{i} x_{t} d v_{i t}+B u_{i} d t & \\
& +\sum_{i=1}^{p} B_{i} u_{i} d v_{i t}+e^{-\alpha t} d w_{t}, t \in[0, \infty),
\end{aligned}
$$

measurements

$$
y_{t}=C x_{t},
$$

and output-feedback law

$$
u_{t}=K y_{t}
$$

the performance criterion

$$
J_{\alpha}(K) \triangleq \lim _{t \rightarrow \infty}\left[e^{2 \alpha t}\left[x_{t}^{T} R_{1} x_{t}+2 x_{t}^{T} R_{12} u_{t}+u_{t}^{T} R_{2} u_{t}\right]\right.
$$

is minimized.

Remark 3.2.1: The exponential time weighting of the disturbance noise in (3.2.1) is required to balance the exponential weighting in the cost (3.2.4). It has no physical significance as such.

To develop necessary conditions for this problem, $K$ must be restricted to the set of second-moment-stabilizing gains

$$
\begin{aligned}
\mathcal{S}_{\alpha} \triangleq\left\{K \in \mathrm{P}^{m \times l}: \tilde{A_{\alpha}} \oplus\right. & \widetilde{A_{\alpha}} \\
& \left.+\sum_{i=1}^{p} \gamma_{i} \tilde{A_{i}} \otimes \tilde{A_{i}} \text { is asymptotically stable }\right\} .
\end{aligned}
$$

For the shifted plant dynamics. The requirement $K \in \mathrm{S}_{\alpha}$ implies the existence of the steady-state nonnegative-definite covariance $Q \triangleq \lim _{t \rightarrow \infty}\left[\sum^{2 \alpha t}\left[x_{t} x_{t}^{T}\right]\right.$. Furthermore, $Q$ is the unique solution to the modified Lyapunov equation

$$
0=\tilde{A}_{\alpha} Q+Q \tilde{A}_{\alpha}^{T}+\sum_{i=1}^{p} \gamma_{i} \tilde{A}_{i} Q \tilde{A}_{i}^{T}+V
$$

An additional technical assumption is that $K$ be confined to the set

$$
\mathcal{S}_{\alpha}^{+} \triangleq\left\{K \in \mathcal{S}_{\alpha}: C Q C^{T}>0 \text {, where } Q \text { satisfies }(3.2 .5)\right\} .
$$

The positive definiteness condition holds, for example, when $Q$ is positive definite and $C$ has full row rank.

Theorem 3.2.1: Suppose $K \in \mathcal{S}^{+}$solves the static optimal control problem. Then these exist $P, Q \in \mathbb{N}^{n}$ such that $K$ is given by

$$
K=-R_{2 s}^{-1} P_{s} Q C^{T}\left(C Q C^{T}\right)^{-1}
$$

and such that $P$ and $Q$ satisfy

$0=A_{\alpha}^{T} P+P A_{\alpha}+\sum_{i=1}^{p} \gamma_{i} A_{i}^{T} P A_{i}+R_{\mathrm{I}}$

$$
-P_{s}^{T} R_{2 s}^{-1} P_{s}+\hat{\tau}_{\perp}^{T} P_{s}^{T} R_{2 s}^{-1} P_{s} \hat{\tau}_{\curlywedge},
$$




$$
\begin{aligned}
0=\left(A_{\alpha}\right. & \left.-B R_{2 s}^{-1} P_{s} \hat{\tau}\right) Q+Q\left(A_{\alpha}-B R_{2 s}^{-1} P_{s} \hat{\tau}\right)^{T} \\
& +\sum_{i=1}^{p} \gamma_{i}\left(A_{i}-B_{i} R_{2 s}^{-1} P_{s} \hat{\tau}\right) Q\left(A_{i}-B_{i} R_{2 s}^{-1} P_{s} \hat{\tau}\right)^{T}+V
\end{aligned}
$$

where

$$
\hat{\tau} \triangleq Q C^{T}\left(C Q C^{T}\right)^{-1} C, \hat{\tau}_{\perp} \triangleq I_{n}-\hat{\tau} .
$$

Proof: First note that from [49] it follows that the exponential factors in (3.2.1) and (3.2.4) are equivalent to replacing $A$ by $A_{\alpha}$. From [57, Theorem 8.5 .5 , p. 142]. it follows that the state covariance $Q(t) \triangleq \Xi^{\xi}\left[e^{2 \alpha t} x_{l} x_{t}^{T}\right]$ satisfies

$$
\dot{Q}(t)=\tilde{A_{\alpha}} Q(t)+Q(t) \tilde{A}_{\alpha}^{T}+\sum_{i=1}^{p} \gamma_{i} \tilde{A_{i}} Q(t) \tilde{A}_{i}^{T}+V .
$$

Since $K \in \mathfrak{S}_{\alpha}, Q \triangleq \lim _{t \rightarrow \infty} Q(t)$ exists, is nonnegative definite, and is the unique solution to (3.2.5). Note that

$$
J_{\alpha}(K)=\operatorname{tr} Q \hat{R} .
$$

Now define the Lagrangian

$$
L(Q, K) \triangleq \operatorname{tr}\left[\lambda Q \tilde{R}+\left(\bar{A}_{\alpha} Q+Q \tilde{A}_{\alpha}^{T}+\sum_{i=1}^{p} \gamma_{i} \tilde{A}_{i} Q \tilde{A}_{i}^{T}+V\right) P\right]
$$

with multipliers $\lambda \geq 0$ and $P \in$ R $^{n \times n}$, and compute

$$
\partial L / \partial Q=\tilde{A}_{\alpha}^{T} P+P \tilde{A_{\alpha}}+\sum_{i=1}^{p} \gamma_{i} \tilde{A}_{i}^{T} P \tilde{A}_{i}+\lambda \tilde{R}
$$

Setting $\partial L / \partial Q=0, \lambda=0$ implies $P=0$ since $K \in \S_{\alpha}$. Hence, without loss of generality set $\lambda=1$ so that

$$
0=\tilde{A}_{\alpha}^{T} P+P \tilde{A}_{\alpha}+\sum_{i=1}^{p} \gamma_{i} \tilde{A}_{i}^{T} P \tilde{A}_{i}+\tilde{R} .
$$

Since $P$ is the (unique) steady-state covariance of the dual system, it is nonnegative definite. Also, since $S_{\alpha}^{+}$is open, evaluating $\partial L /$ $\partial K=0$ yields

$$
0=R_{2 s} K C Q C^{T}+P_{s} Q C^{T} .
$$

Since $K \in \mathrm{s}_{\alpha}^{+}, C Q C^{T}$ is invertible, and hence (3.2.6) holds. Finally, (3.2.8) is equivalent to (3.2.5) and, performing some algebraic manipulation, (3.2.7) is equivalent to (3.2.10).

Remark 3.2.2: Because $R_{2 s} \geq R_{2}$, and thus $R_{2 s}^{-1} \leq R_{2}^{-1}$, the gain $K$ in (3.2.6) leads to a net decrease in controller authority due to the control-dependent noise. For problems which are open-loop stable and which remain stable under plant variations, this is an intuitively expected consequence of parameter uncertainty.

Remark 3.2.3: Theorem 3.2.1 generalizes and unifies several previous results. In particular, the noise correlation and output feedback constraint constitute generalizations of [26]-[33]. Furthermore, because of the presence of multiplicative noise, the results of [58], [59] are extended. The role of the oblique projection $\hat{\tau}$ has been discussed in [58], [59]. Connections with the oblique projection $\tau$ arising in the dynamic-compensation problem [14] are discussed in [60].

\section{Sufficiency Meets Necessity: A Marriage of the Deterministic and Stochastic}

We now answer our main question: Can a feedback law predicated on a stochastic multiplicative noise model provide guaranteed deterministic robust asymptotic stability? The answer is "yes" provided the exponential is of sufficient magnitude.
Theorem 3.3.1: Suppose there exists $P \in \rho^{n}$ and $Q \in \mathrm{N}^{n}$ satisfying $C Q C^{T}>0,(3.2 .7)-(3.2 .9)$ and

$$
0<\left(2 \alpha-\sum_{i=1}^{p} \delta_{i}^{2} / \gamma_{i}\right) P+\tilde{R}
$$

where $K$ in $\tilde{R}$ is given by (3.2.6). Then $K$ solves the static robust stabilization problem.

Proof: In Theorem 3.1.1 define

$$
\begin{gathered}
\Phi(P)=2 \alpha P+\sum_{i=1}^{p} \gamma_{i} \tilde{A}_{i}^{T} P \tilde{A_{i}}+\tilde{R} \\
M_{i}=\left(\gamma_{i} / \delta_{i}\right)^{1 / 2} P^{1 / 2} \tilde{A}_{i}, N_{i}=\left(\delta_{i} / \gamma_{i}\right)^{1 / 2} P^{1 / 2} .
\end{gathered}
$$

Note that (3.1.5)-(3.1.8) hold. Furthermore, because of the equivalence of (3.2.10) and (3.2.7), it follows that (3.1.9) is equivalent to (3.2.7). Finally, $(3.1 .10)$ is a consequence of (3.3.1).

Remark 3.3.1: Note that (3.2.7)-(3.2.9) serve to construct a Lyapunov function guaranteeing robust stability. Hence, it is not necessary to actually verify that $K \in \mathcal{S}_{\alpha}^{+}$.

By strengthening (3.3.1) the following simplification is immediate.

Corollary 3.3.1: Suppose there exists $P \in P^{n}$ and $Q \in \mathbb{N}^{n}$ satisfying $C Q C^{T}>0$, (3.2.7)-(3.2.9) and

$$
\alpha>\frac{1}{2} \sum_{i=1}^{p} \sigma_{i}^{2} / \gamma_{i}
$$

Then $K$ given by $(3.2 .6)$ solves the static robust stabilization problem.

It is interesting to note that the feedback gain given by Corollary 3.3.1 may be an extremal, i.e., local minimum, local maximum, etc., and not necessarily a solution of the static optimal control problem. The result is valid, however, for all extremals of the optimization problem. By specializing Corollary 3.3.1 to a solution, i.e., global minimum, of the optimal control problem, we can bridge the gap between sufficiency and necessity.

Corollary 3.3.2: Suppose $K \in \mathcal{S}_{\alpha}^{+}$solves the static optimal control problem where $\alpha$ satisfies (3.3.1), and suppose that the corresponding solution $P$ of (3.2.7) is positive definite. Then $K$ also solves the static robust stabilization problem.

\section{Dynamic Output FeedBack}

\section{A. Dynamic Robust Stabilization Problem: Deterministic Sufficiency Theory}

Consider the following problem.

Dynamic Robust Stabilization Problem: Determine $\left(A_{c}, B_{c}\right.$, $C_{c}$ ) such that the closed-loop system consisting of the controlled plant (3.1.1), measurements

$$
y(t)=\left(C+\sum_{i=1}^{p} \sigma_{i}(t) C_{i}\right) x(t)
$$

and dynamic output-feedback law

$$
\begin{gathered}
\dot{x}_{c}(t)=A_{c} x_{c}(t)+B_{c} y(t), \\
u(t)=C_{c} x_{c}(t)
\end{gathered}
$$

is asymptotically stable for all measurable $\left(\sigma_{1}, \cdots, \sigma_{p}\right):[0, \infty) \rightarrow$ $\mathrm{R}^{p}$ satisfying (3.1.4).

Remark 4.1.1: Note that the problem statement places no restriction on the order $n_{c}$ of the dynamic compensator. Also, we now permit uncertainties in the observation matrix $C$ by including perturbations $\sigma_{i}(t) C_{i}$ in $(4.1 .1)$. 
The following result is completely analogous to Theorem 3.1.1. Theorem 4.1.1: Given $\left(A_{c}, B_{c}, C_{c}\right)$ assume there exist

$$
\begin{aligned}
& \tilde{\Phi}: \mathfrak{p}^{\hat{n}} \rightarrow \mathfrak{S}^{\tilde{n}}, \\
& \tilde{P} \in \mathrm{\rho}^{\tilde{n}}, \\
& \tilde{M}_{i}, \tilde{N}_{i} \in \tilde{N}^{\tilde{n}_{i} \times \tilde{n}}, \quad i=1, \cdots, p
\end{aligned}
$$

such that

$$
\begin{gathered}
\tilde{M}_{i}^{T} \tilde{N}_{i}+\tilde{N}_{i}^{T} \tilde{M}_{i}=\tilde{\tilde{A}}_{i}^{T} \tilde{P}+\tilde{P} \tilde{\tilde{A}}, \quad i=1, \cdots, p, \\
0=\tilde{\tilde{A}}^{r} \tilde{P}+\tilde{P} \tilde{\tilde{A}}+\tilde{\Phi}(\tilde{P}), \\
\sum_{i=1}^{p} \delta_{i}\left(\tilde{M}_{i}^{T} \tilde{M}_{i}+\tilde{N}_{i}^{T} \tilde{N}_{i}\right)<\tilde{\Phi}(\tilde{P}) .
\end{gathered}
$$

Then $\left(A_{c}, B_{c}, C_{c}\right)$ solves the dynamic robust-stabilization problem.

\section{B. Dynamic Optimal Control Problem: Stochastic Necessity Theory}

We now consider the dynamic optimal control problem with state-, control-, and measurement-dependent white noise and exponentially weighted quadratic cost. The optimization is performed over the class of dynamic compensators of fixed order $n_{c}$ $\leq n$.

Dynamic Optimal Control Problem: Determine $\left(A_{\mathfrak{c}}, B_{c}, C_{c}\right)$ such that, for the closed-loop system consisting of the controlled plant

$$
\begin{aligned}
d x_{t}=A x_{t} d t+\sum_{i=1}^{p} A_{i} x_{t} d v_{i t}+B u_{i} d t & \\
& +\sum_{i=1}^{p} B_{i} u_{t} d v_{i t}+e^{-\alpha t} d w_{1 l}, t \in[0, \infty)
\end{aligned}
$$

measurements

$$
d y_{t}=C x_{\imath} d t+\sum_{i=1}^{p} C_{i} x_{t} d v_{i t}+e^{-\alpha t} d w_{2 t}
$$

and dynamic output-feedback law

$$
\begin{gathered}
d x_{c t}=A_{c} x_{c t} d t+B_{c} d y_{t}, \\
u_{t}=C_{c} x_{c t}
\end{gathered}
$$

the performance criterion

$$
\begin{aligned}
J_{\alpha}\left(A_{c}, B_{c}, C_{c}\right) \doteq \lim _{t \rightarrow \infty} 3 e^{2 \alpha t}\left[x_{i}^{T} R_{1} x_{t}\right. & \\
& \left.+2 x_{t}^{T} R_{12} u_{t}+u_{t}^{T} R_{2} u_{t}\right]
\end{aligned}
$$

is minimized.

To develop necessary conditions we restrict $\left(A_{c}, B_{c}, C_{c}\right)$ to the set

$D_{\alpha} \triangleq\left\{\left(\tilde{\tilde{A}_{c}}, B_{c}, C_{c}\right): \overline{\tilde{A}}_{\sim} \oplus \tilde{\overline{A_{\alpha}}}+\sum_{i=1}^{p} \gamma_{i} \tilde{\widetilde{A}}_{i} \otimes \tilde{\tilde{A}}_{i}\right.$ is

asymptotically stable and $\left(A_{c}, B_{c}, C_{c}\right)$ is minimal $\}$

and invoke the technical assumption

$$
\left[B_{i} \neq 0 \Rightarrow C_{i}=0\right], \quad i=1, \cdots, p .
$$

The following lemma will be needed.
Lemma 4.2.1: If $\hat{Q}, \hat{P} \in \mathrm{N}^{n}$ and rank $\hat{Q} \hat{P}=n_{c}$, then there exist $G, \Gamma \in \mathbb{R}^{n^{n}}{ }^{\times n}$ and invertible $M \in \mathbb{R}^{n}{ }^{n} \times n_{c}$ such that

$$
\begin{gathered}
\hat{Q P}=G^{T} M \Gamma, \\
\Gamma G^{T}=I_{n_{\mathcal{C}^{*}}}
\end{gathered}
$$

Furthermore, $G, M$, and $\Gamma$ are unique modulo a change of basis in $\mathbb{B}^{n}{ }^{\times} \times n_{c}$.

Proof: The result is an immediate consequence of $[61$, Theorem 6.2.5, p. 123].

Note that because of (4.2.8), the $n \times n$ matrix $\tau=G^{T} \Gamma$ is idempotent, i.e., $\tau^{2}=\tau$. Since $\tau$ is not necessarily symmetric, it is an oblique projection.

Theorem 4.2.1: Suppose (4.2.6) holds and $\left(A_{\mathcal{c}}, B_{c}, C_{c}\right) \in \mathbb{D}_{\alpha}$ solves the dynamic optimal control problem. Then there exist $Q$, $P, \hat{Q}, \hat{P} \in \mathbb{N}^{n}$ such that $A_{c}, B_{c}, C_{c}$ are given by

$$
\begin{gathered}
A_{c}=\Gamma\left(A-B R_{2 d}^{-1} P_{d}-Q_{d} V_{2 d}^{-1} C\right) G^{T}, \\
B_{c}=\Gamma Q_{d} V_{2 d}^{-1}, \\
C_{c}=-R_{2 d}^{-1} P_{d} G^{T}
\end{gathered}
$$

and such that $Q, P, \hat{Q}, \hat{P}$ satisfy

$$
\begin{aligned}
0=A_{\alpha} Q & +Q A_{\alpha}^{T}+V_{\mathrm{I}}+\sum_{i=1}^{p} \gamma_{i}\left[A_{i} Q A_{i}^{T}+\left(A_{i}-B_{i} R_{2 d}^{-1} P_{d}\right) \hat{Q}\left(A_{i}\right.\right. \\
\left.-B_{i} R_{2 d}^{-1} P_{d}\right)^{T} & -Q_{d} V_{2 d}^{-1} Q_{d}^{T}+\tau_{\perp} Q_{d} V_{2 d}^{-1} Q_{d}^{T} \tau_{\perp}^{T},
\end{aligned}
$$

$$
0=A_{\alpha}^{T} P+P A_{\alpha}+R_{1}+\sum_{i=1}^{p} \gamma_{i}\left[A_{i}^{T} P A_{i}+\left(A_{i}-Q_{d} V_{2 d}^{-1} C_{i}\right)^{T} \hat{P}\left(A_{i}\right.\right.
$$$$
\left.\left.-Q_{d} V_{2 d}^{-1} C_{i}\right)\right]-P_{d}^{T} R_{2 d}^{-1} P_{d}+\tau_{\perp}^{T} P_{d}^{T} R_{2 d}^{-1} P_{d} \tau_{\perp},
$$$$
0=A_{P \alpha} \hat{Q}+\hat{Q} A_{P \alpha}^{T}+Q_{d} V_{2 d}^{-1} Q_{d}^{T}-\tau_{\perp} Q_{d} V_{2 d}^{-1} Q_{d}^{T} \tau_{\perp}^{T} \text {, }
$$

$$
0=A_{Q \alpha}^{T} \hat{P}+\hat{P} A_{Q \alpha}+P_{d}^{T} R_{2 d}^{-1} P_{d}-\tau_{\perp}^{T} P_{d}^{T} R_{2 d}^{-1} P_{d} \tau_{\perp}
$$$$
\operatorname{rank} \hat{Q}=\operatorname{rank} \hat{P}=\operatorname{rank} \hat{Q} \hat{P}=n_{c}
$$

(4.2.7) and (4.2.8), where

$$
\tau \triangleq G^{T} \Gamma, \tau_{\perp} \triangleq I_{n}-\tau .
$$

Proof: As in the proof of Theorem 3.2.1 we note that the exponential factors in (4.2.1), (4.2.2), and (4.2.5) are equivalent to replacing $A$ and $A_{c}$ by $A+\alpha I_{n}$ and $A_{c}+\alpha I_{n_{\mathrm{c}}}$, respectively. Theorem 4.2.1 now follows immediately from [40, Theorem 2.3]. It need only be noted that (4.2.9) follows from

$$
A_{c}+\alpha I_{n_{c}}=\Gamma\left(A_{\alpha}-B R_{2 d}^{-1} P_{d}-Q_{d} V_{2 d}^{-1} C\right) G^{T}
$$

and the fact that $\Gamma A_{\alpha} G^{T}=\Gamma A G^{T}+\alpha I_{n_{c}}$ because of (4.2.8).

\section{Sufficiency Meets Necessity: The Dynamic Case}

We now bridge the gap between sufficiency and necessity for dynamic controllers.

Theorem 4.3.1: Assume (4.2.6) holds and suppose there exists $Q, P, \hat{Q}, \hat{P} \in \mathbb{N}^{n}$ satisfying (4.2.12)-(4.2.17), (4.2.7), (4.2.8),

$$
\tilde{P} \triangleq\left[\begin{array}{cc}
P+\hat{P} & -\hat{P} G^{T} \\
-G \hat{P} & G \hat{P} G^{T}
\end{array}\right]>0
$$


and

$$
0<\left(2 \alpha-\sum_{i=1}^{p} \delta_{i}^{2} / \gamma_{i}\right) \tilde{P}+\tilde{\tilde{R}}
$$

Then $\left(A_{c}, B_{c}, C_{c}\right)$ given by (4.2.9)-(4.2.11) solves the dynamic robust stabilization problem.

Remark 4.3.1: Note that $\tilde{P}$ is always at least nonnegative definite since

$$
\tilde{P}=\left[\begin{array}{ll}
P & 0 \\
0 & 0
\end{array}\right]+\left[\begin{array}{c}
\hat{P}^{1 / 2} \\
-G \hat{P}^{1 / 2}
\end{array}\right]\left[\begin{array}{c}
\hat{P}^{1 / 2} \\
-G \hat{P}^{1 / 2}
\end{array}\right]^{T} \geq 0 .
$$

Proof of Theorem 4.3.1: As shown in [40], (4.2.6)(4.2.17) are equivalent to

$$
\begin{aligned}
& 0=\tilde{\tilde{A}}_{\alpha}^{T} \tilde{P}+\tilde{P} \tilde{\tilde{A}}+\sum_{i=1}^{p} \gamma_{i} \tilde{\tilde{A}}_{i}^{T} \tilde{P} \overline{\tilde{A}_{i}}+\tilde{\bar{R}}, \\
& 0=\tilde{\tilde{A}} \alpha \tilde{Q}+\tilde{Q} \tilde{\bar{A}}_{\alpha}^{T}+\sum_{i=1}^{p} \gamma_{i} \overline{\tilde{A}}_{i} \tilde{Q} \tilde{\bar{A}}_{i}^{T}+\tilde{\tilde{V}}
\end{aligned}
$$

where

$$
\tilde{Q}=\left[\begin{array}{cc}
Q+\hat{Q} & \hat{Q} \Gamma^{T} \\
\Gamma \hat{Q} & \Gamma \hat{Q} \Gamma^{T}
\end{array}\right]
$$

The result now follows from Theorem 4.1.1 as in the proof of Theorem 3.3.1.

Remark 4.3.2: As in Corollary 3.3.1 the inequality (4.3.2) can be replaced by the stronger condition (3.3.2).

\section{The Petersen-Hollot Approach to Robust Stabilization}

\section{A. Static Output Feedback}

The deterministic Riccati equation approach of Petersen and Hollot is based upon factoring $A_{i}$ and $B_{i}$ as

$$
A_{i}=D_{i} E_{i}, B_{i}=D_{i} F_{i}, \quad i=1, \cdots, p
$$

where $D_{i} \in \mathbb{R}^{n \times n_{i}}, E_{i} \in \mathbb{R}^{n_{i} \times n}$, and $F_{i} \in \mathbb{R}^{n_{i} \times m}$. Obviously, such a factorization may not be unique, and the nonuniqueness is an element of the sufficiency test. To state the sufficiency condition we shall require the notation

$$
\begin{gathered}
R_{2 a} \triangleq R_{2}+\sum_{i=1}^{p} \delta_{i} F_{i}^{T} F_{i}, P_{a} \triangleq B^{T} P+R_{12}^{T}+\sum_{i=1}^{p} \delta_{i} F_{i}^{T} E_{i}, \\
Q_{a}=Q C^{T}+V_{12}, D \triangleq \sum_{i=1}^{p} \delta_{i} D_{i} D_{i}^{T}, E \triangleq \sum_{i=1}^{p} \delta_{i} E_{i}^{T} E_{i} .
\end{gathered}
$$

Theorem 5.1.1: Assume there exist $P \in \mathrm{P}^{n}$ and $Q \in \mathbb{N}^{n}$ satisfying $C Q C^{T}>0$,

$$
0=A^{T} P+P A+P D P+R_{1}+E-P_{a}^{T} R_{2 a}^{-1} P_{a}+\hat{\tau}_{\perp}^{T} P_{a}^{T} R_{2 a}^{-1} P_{a} \hat{\tau}_{\perp},
$$

$$
0=\left(A+D P-B R_{2 a}^{-1} P_{a} \hat{\tau}\right) Q+Q\left(A+D P-B R_{2 a}^{-1} P_{a} \hat{\tau}\right)^{T}+V
$$

and

$$
\tilde{R}>0
$$

where $\hat{\tau}$ is given by (3.2.9) and $K$ in $\tilde{R}$ is given by

$$
K=-R_{2 a}^{-1} P_{a} Q C^{T}\left(C Q C^{T}\right)^{-1} .
$$

Proof: In Theorem 3.1.1 define

$$
\begin{gathered}
\Phi(P)=\tilde{R}+\sum_{i=1}^{p} \delta_{i}\left[\left(E_{i}+F_{i} K C\right)^{T}\left(E_{i}+F_{i} K C\right)+P D_{i} D_{i}^{T} P\right], \\
M_{i}=E_{i}+F_{i} K C, N_{i}=D_{i}^{T} P
\end{gathered}
$$

so that (3.1.5)-(3.1.8) hold. Note that (5.1.2) and (5.1.3) are equivalent to (3.1.9). Finally, because of $(5.1 .4),(3.1 .10)$ is satisfied.

Remark 5.1.1: Theorem 5.1.1 generalizes [25, Theorem 3.3] in four distinct ways. First, in [25], the uncertainties $A$ and $B$ are assumed to be independent. In our setup this corresponds to the additional condition that $A_{i} \neq 0$ implies $B_{i}=0$. In this case (5.1.1) is satisfied with either $E_{i}=0$ or $F_{i}=0$ for each $i$. Second, in [25], $A_{i}$ and $B_{i}$ are confined to have unit rank. This would be the case if we required $n_{i}=1$ in (5.1.1). Third, in [25], $R_{12}=0$. And, fourth, the results of [25] apply only to the statefeedback case, i.e., $C=I_{n}$. In this case (5.1.3) is superfluous and $\hat{\tau}=I_{n}$.

\section{B. Dynamic Output Feedback}

We now extend the Petersen-Hollot approach to reduced-order dynamic compensation. Our only constraint is that we do not permit uncertainty in the observation matrix. Define

$$
A_{P} \triangleq A-B R_{2 a}^{-1} P_{a}, A_{Q} \triangleq A-Q_{a} V_{2}^{-1} C .
$$

Theorem 5.2.1: Assume $C_{1}=\cdots=C_{p}=0$ and suppose there exist $Q, P, \hat{Q}, \hat{P} \in \mathbb{N}^{n}$ satisfying

$$
\begin{aligned}
0=[A+D(P+\hat{P})] Q & +Q[A+D(P+\hat{P})]^{T} \\
& +V_{1}-Q_{a} V_{2}^{-1} Q_{a}^{T}+\tau_{\perp} Q_{a} V_{2}^{-1} Q_{a}^{T} \tau_{\perp}^{T},
\end{aligned}
$$

$$
0=\left(A_{P}+D P\right) \hat{Q}+\hat{Q}\left(A_{P}+D P\right)^{T}+Q_{a} V_{2}^{-1} Q_{a}^{T}-\tau_{\perp} Q_{a} V_{2}^{-1} Q_{a}^{T} \tau_{\perp}^{T}
$$

$0=\left(A_{Q}+D P\right)^{T} \hat{P}+\hat{P}\left(A_{Q}+D P\right)+\hat{P} D \hat{P}$

$$
+P_{a}^{T} R_{2 a}^{-1} P_{a}-\tau_{\perp}^{T} P_{a}^{T} R_{2 a}^{-1} P_{a} \tau_{\perp},
$$

(4.2.7), (4.2.8), (4.2.16), (4.3.1), and

$$
\left[\begin{array}{cc}
R_{1} & -R_{12} R_{2 a}^{-1} P_{a} G^{T} \\
-G P_{a}^{T} R_{2 a}^{-1} R_{12}^{T} & G P_{a}^{T} R_{2 a}^{-1} R_{2} R_{2 a}^{-1} P_{a} G^{T}
\end{array}\right]>0 .
$$

Then $\left(A_{c}, B_{c}, C_{c}\right)$ given by

$$
\begin{gathered}
A_{\mathrm{c}}=\Gamma\left(A-Q_{a} V_{2}^{-1} C-B R_{2 a}^{-1} P_{a}+D P\right) G^{T}, \\
B_{c}=\Gamma Q_{a} V_{2}^{-1}, \\
C_{\mathrm{c}}=-R_{2 a}^{-1} P_{a} G^{T}
\end{gathered}
$$

solves the dynamic robust stabilization problem.

Proof: In Theorem 4.1.1 define

$$
\tilde{\Phi}(\tilde{P})=\tilde{R}+\sum_{i=1}^{p} \delta_{i}\left(\tilde{\tilde{E}}_{i}^{T} \tilde{\tilde{E}}_{i}+\tilde{P} \tilde{\tilde{D}}_{i} \tilde{\bar{D}}_{i}^{T} \tilde{P}\right), \tilde{M}_{i}=\tilde{\tilde{E}}_{i}, \tilde{N}_{i}=\tilde{D}_{i}^{T} \tilde{P},
$$

$$
\tilde{\overline{D_{i}}}=\left[\begin{array}{cc}
D_{i} & 0 \\
0 & 0
\end{array}\right], \tilde{E_{i}}=\left[\begin{array}{cc}
E_{i} & F_{i} C_{c} \\
0 & 0
\end{array}\right]
$$


and note that (5.2.2) and (5.2.4) correspond to (4.1.8). Also, note that $(5.2 .1)$ and $(5.2 .3)$ correspond to

$0=\left(\tilde{\tilde{A}}+\sum_{i=1}^{p} \delta_{i} \tilde{\tilde{D}}_{i} \tilde{\bar{D}}_{i}^{T} \tilde{P}\right) \tilde{Q}+\tilde{Q}\left(\tilde{\bar{A}}+\sum_{i=1}^{p} \delta_{i} \tilde{\tilde{D}}_{i} \tilde{\tilde{D}}_{i}^{T} \tilde{P}\right)^{T}+\tilde{\tilde{V}}$

Remark 5.2.1: Theorem 5.2.1 was discovered by optimizing $J$ $=\operatorname{tr} \tilde{P} \tilde{V}$ subject to

$$
0=\tilde{\tilde{A}}^{T} \tilde{P}+\tilde{P} \tilde{\tilde{A}}+\tilde{\tilde{R}}+\sum_{i=1}^{p} \delta_{i}\left[\tilde{E}_{i}^{T} \tilde{\tilde{E}}_{i}+\tilde{P} \tilde{\tilde{D}}_{i} \tilde{\tilde{D}}_{i}^{T} \tilde{P}\right]
$$

and using the techniques of [40], [41]. As shown in [54], this approach also yields robust performance bounds.

\section{ACKNOWLEDGMENT}

The author wishes to thank Prof. C. V. Hollot for numerous helpful discussions and for carefully reviewing the original manuscript of the paper.

\section{REFERENCES}

[1] P. K. Wong and M. Athans, "Closed-loop structural stability for linear-quadratic optimal systems," IEEE Trans. Automat. Contr., vol. AC-22, pp. 94-99, 1977.

[2] M. G. Safonov and M. Athans, "Gain and phase margin for multiloop LQG regulators," IEEE Trans. Automat. Contr., vol. AC-22, pp. $173-179,1977$.

[3] J. C. Doyle, "Guaranteed margins for LQG regulators," IEEE Trans. Automat. Contr., vol. AC-23, pp. 756-757, 1978.

[4] J. C. Doyle and G. Stein, "Robustness and observers," IEEE Trans. Automat. Contr., vol. AC-24, pp. 607-611, 1979.

[5] - "Multivariable feedback design: Concepts for a classical modern synthesis," IEEE Trans. Automat. Contr, vol. AC-26, pp. $4-16,1981$.

[6] G. Stein and M. Athans, "The LQG/LTR procedure for multivariable feedback control design," IEEE Trans. Automat. Contr., vol. AC32, pp. 105-114, 1987.

[7] E. Soroka and U. Shaked, "On the robustness of LQ regulators," IEEE Trans. Automat. Contr., vol. AC-29, pp. 664-665, 1984.

[8] U. Shaked and E. Soroka, "On the stability robustness of the continuous-time LQG optimal control," IEEE Trans. Automat. Contr., vol. AC-30, pp. 1039-1043, 1985.

[9] M. Tahk and J. L. Speyer, "Modeling of parameter variations and asymptotic LQG synthesis," in Proc. 25th IEEE Conf. Decision Contr., Athens, Greece, Dec. 1986, pp. 1459-1465.

[10] S. S. L. Chang and T. K. C. Peng, "Adaptive guaranteed cost control of systems with uncertain parameters," IEEE Trans. Automat. Contr., vol. AC-17, pp. 474-483, 1972

[11] R. V. Patel, M. Toda, and S. Sridhar, "Robustness of linear quadratic state feedback designs in the presence of system uncertainty," IEEE Trans. Automat. Contr., vol. AC-22, pp. 945-949, 1977.

[12] A. M. Meilakhs, "Design of stable control systems subject to parametric perturbation," Automat. Remote Contr., pp. 1409-1418, 1979.

[13] A. Vinkler and L. J. Wood, "Multistep guaranteed cost control of linear systems with uncertain parameters," J. Guid. Contr., vol. 2, pp. $449-456,1979$.

[14] M. Eslami and D. L. Russell, "On stability with large parameter variations: Stemming from the direct method of Lyapunoy," IEEE Trans. Automat. Contr., vol. AC-25, pp. 1231-1234, 1980.

[15] M. Corless and G. Leitmann, "Continuous state feedback guaranteeing uniform ultimate boundedness for uncertain dynamical systems," IEEE Trans. Automat. Contr., vol. AC-26, pp. 1139-1144, 1981

[16] J. S. Thorp and B. R. Barmish, "On guaranteed stability of uncertain linear systems via linear control," J. Optimiz. Theory Appl., vol. 35, pp. 559-579, 1981.

[17] E. Noldus, "Design of robust state feedback laws," Int. J. Contr., vol. 35 , pp. 935-944, 1982.

[18] B. R. Barmish, I. R. Petersen, and A. Feuer, "Linear ultimate boundedness control of uncertain dynamic systems," Automatica, vol. 19 , pp. 523-532, 1983.

[19] B. R. Barmish, M. Corless, and G. Leitmann, "A new class of stabilizing controllers for uncertain dynamical systems," SIAM $J$. Contr. Optimiz., vol. 21, pp. 246-255, 1983.

[20] I. R. Petersen and B. R. Barmish, "Control effort considerations in the stabilization of uncertain dynamical systems," in Proc. Amer. Contr. Conf., San Diego, CA, June 1984, pp. 490-495.
[21] B. R. Barmish, "Necessary and sufficient conditions for quadratic stabilizability of an uncertain system," J. Optimiz. Theory Appl., vol. 46 , pp. $399-408,1985$

[22] R. K. Yedavalli, S. S. Banda, and D. B. Ridgely, "Time-domain stability robustness measures for linear regulators," $J$. Guidance Contr. Dynam., vol. 8, pp. 520-524, 1985.

[23] I. R. Petersen, "A Riccati equation approach to the design of stabilizing controllers and observers for a class of uncertain linear systems," IEEE Trans. Automat. Contr., vol. AC-30, pp. 904-907, 1985.

[24] A. R. Galimidi and B.R. Barmish, "The constrained Lyapunov problem and its application to robust output feedback stabilization," IEEE Trans. Automat. Contr., vol. AC-31, pp. 410-419, 1986.

[25] I. R. Petersen and C. V. Hollot, "A Riccati equation approach to the stabilization of uncertain systems," Automatica, vol. 22, pp. 397$411,1986$.

[26] W. M. Wonham, "Optimal stationary control of a linear system with state-dependent noise," SIAM J. Contr., vol. 5, pp. 486-500, 1967.

[27] _- - "On a matrix Riccati equation of stochastic control," SIAM J. Contr., vol. 6, pp. 681-697, 1968.

[28] D. Kleinman, "Optimal stationary control of linear systems with control-dependent noise," IEEE Trans. Automat. Contr., vol. AC14, pp. 673-677, 1969.

[29] P. McLane, "Optimal stochastic control of linear systems with state and control-dependent disturbances," IEEE Trans. Automat. Contr., vol. AC-16, pp. 793-798, 1971.

[30] P. J. McLane, "Linear optimal stochastic control using instantaneous output feedback," Int. J. Contr., vol. 13, pp. 383-396, 1971.

[31] U. Haussmann, "Optimal stationary control with state and control dependent noise," SIAM J. Contr., vol. 9, pp. 184-198, 1971.

[32] C. W. Merriam, III, Automated Design of Control Systems. New York: Gordon and Breach, 1974.

[33] J. Bismut, "Linear-quadratic optimal stochastic control with random coefficients," SIAM J. Contr., vol. 14, pp. 419-444, 1976.

[34] G. N. Mil'stein, "Design of stabilizing controller with incomplete state data for linear stochastic system with multiplicative noise," Automat. Remote Contr., vol. 43, pp. 653-659, 1982.

[35] Y. A. Phillis, "Controller design of systems with multiplicative noise," IEEE Trans. Automat. Contr., vol. AC-30, pp. 1017-1019, 1985.

[36] D. S. Bernstein and D. C. Hyland, "Optimal projection/maximum entropy stochastic modelling and reduced-order design synthesis," in Proc. IFAC Workshop on Model Error Concepts and Compensation, Boston, MA, June 1985, pp. 47-54.

[37] ___ "The optimal projection/maximum entropy approach to designing low-order, robust controllers for flexible structures," in Proc. 24ih IEEE Conf. Decision Contr., Fort Lauderdale, FL, Dec. 1985, pp. 745-752.

[38] D. S. Bernstein, L. D. Davis, S. W. Greeley, and D. C. Hyland, "Numerical solution of the optimal projection/maximum entropy design equations for low-order, robust controller design," in Proc. 24th IEEE Conf. Decision Contr., Fort Lauderdale, FL, Dec. 1985, pp. 1795-1798.

[39] D. S. Bernstein and S. W. Greeley, "Robust controller synthesis using the maximum entropy design equations," IEEE Trans. Automat. Contr., vol. AC-31, pp. 362-364, 1986.

[40] D. S. Bernstein and D. C. Hyland, "The optimal projection equations for reduced-order modelling, estimation and control of linear systems with multiplicative white noise," J. Optimiz. Theory Appl., 1988.

[41] D. C. Hyland and D. S. Bernstein, "The optimal projection equations for fixed-order dynamic compensation," IEEE Trans. Automat. Contr., vol. AC-29, pp. 1034-1037, 1984.

[42] P. J. McLane, "Asymptotic stability of linear autonomous systems with state-dependent noise," IEEE Trans. Automat. Contr., vol. AC-14, pp. 754-755, 1969 .

[43] J. L. Willems, "Mean square stability criteria for stochastic feedback systems," Int. J. Syst. Sci., vol. 4, pp. 545-564, 1973.

[44] U. G. Haussmann, "Stability of linear systems with control dependent noise," SIAM J Contr., vol. 11, pp. 382-394, 1973.

[45] U. Haussmann, "On the existence of moments of stationary linear systems with multiplicative noise," SIAM J. Contr., vol. 12, pp. 99105,1974 .

[46] J. L. Willems and J. C. Willems, "Feedback stabilizability for stochastic systems with state and control dependent noise," Automatica, vol. 12, pp. 277-283, 1976.

[47] J. L. Willems and J. C. Willems, "Robust stabilization of uncertain systems," SIAM J. Contr. Optimiz., vol. 21, pp. 352-374, 1983.

[48] L. Arnold, H. Crauel, and V. Wihstutz, "Stabilization of linear systems by noise," SIAM J. Contr. Optimiz., vol. 21, pp. 451-461, 1983.

[49] B. D. O. Anderson and J. B. Moore, Linear Optimal Control. Englewood Cliffs, NJ: Prentice-Hall, 1970

[50] M. Athans, R. T. Ku, and S. B. Gershwin, "The uncertainty threshold principle: Some fundamental limitations of optimal decision making under dynamic uncertainty," IEEE Trans. Automat. Contr., vol. AC-22, pp. 491-495, 1977

[51] R. T. Ku and M. Athans, "Further results on the uncertainty threshold 
principle," IEEE Trans. Automat. Contr., vol. AC-22, pp. 866$868,1977$.

[52] D. S. Bernstein and D. C. Hyland, "Optimal projection for uncertain systems (OPUS): A unified theory of reduced-order, robust control design," in Large Space Structures: Dynamics and Control, S. N. Atluri and A. K. Amos, Eds. New York: Springer-Verlag, 1987.

[53] D. C. Hyland and D. S. Bernstein, "The majorant Lyapunov equation: A nonnegative matrix equation for guaranteed robust stability and performance of large scale systems," Amer. Contr. Conf., Minneapolis, MN, June 1987, pp. 910-917; also in IEEE Trans. Automat. Contr., vol. AC-32, pp. 1005-1013, Nov. 1987.

[54] D. S. Bernstein and W. M. Haddad, "The optimal projection equations with Petersen-Hollot bounds: Robust controller synthesis with guaranteed structured stability radius," IEEE Conf. Decision Contr., Los Angeles, CA, Dec. 1987.

[55] W. M. Haddad, "Robust optimal projection control-system synthesis," Ph.D. dissertation, Dep. Mechanical Eng., Florida Inst. Technol., Melbourne, FL, Mar. 1987.

[56] J. W. Brewer, "Kronecker products and matrix calculus in system theory," IEEE Trans. Circuits Syst., vol. CAS-25, pp. 772-781, 1978.
[57] L. Arnold, Stochastic Differential Equations: Theory and Applications. New York: Wiley, 1974.

[58] J. Medanic " On stabilization and optimization by output feedback," in Proc. Twelfth Asilomar Conf. Circ., Syst. Comp., 1978, pp. 412416.

[59] S. Renjen and D. P. Looze, "Synthesis of decentralized output/state regulators," in Proc. Amer. Contr. Conf., Arlington. VA, June 1982, pp. $758-762$

[60] D. S. Bernstein, "The optimal projection equations for static and dynamic output feedback: The singular case," this issue, pp. 11391143.

[61] C. R. Rao and S. K. Mitra, Generalized Inverse of Matrices and Its Applications. New York: Wiley, 1971.

[62] W. M. Wonham, Linear Multivariable Control: A Geometric Approach. New York: Springer-Verlag. 1979.

Dennis S. Bernstein (M'82), for a photograph and biography, see p. 1013 of the November 1987 issue of this TRANSACTIONS. 